\title{
EL MODELO COGNITIVO DE EVALUACIÓN Y SU INFLUENCIA EN EL MEJORAMIENTO DEL APRENDIZAJE SIGNIFICATIVO DE LOS ALUMNOS DE LA ESCUELA DE EDUCACIÓN DE LA UNIVERSIDAD NACIONAL SANTIAGO ANTÚNEZ DE MAYOLO
}

\author{
THE COGNITIVE MODEL OF EVALUATION AND HIS INFLUENCE IN THE \\ IMPROVEMENT OF THE SIGNIFICANT LEARNING OF THE PUPILS OF THE SCHOOL \\ OF EDUCATION OF THE NATIONAL UNIVERSITY SANTIAGO ANTÚNEZ DE MAYOLO
}

Jesús Manuel More López ${ }^{1}$, Félix Julian Valerio Haro', Víctor Manuel Zegarra Chauca',

Yasmín Jesús Vélez Chang ${ }^{2}$, José Antonio Legua Cárdenas ${ }^{2}$, Antonio Salomón Valderrama Romero ${ }^{3}$

RESUMEN

Objetivo: Plantear el modelo cognitivo de evaluación como un recurso que posibilite el mejoramiento del aprendizaje significativo de estudiantes de la Escuela Profesional De Educación de la Universidad Nacional "Santiago Antunez de Mayolo - Filial Barranca. Material y Métodos: Se aplico un diseño cuasi experimental, empleando el método analítico y síntesis, se tuvo como instrumento a la Escala de Estrategia de Aprendizaje Contextualizado (ESEAC) como esquema. la muestra estratificada fue de 40 estudiantes divididas en 2 grupos, uno de control y el otro de experimento. Resultado: Después de la aplicación del instrumento se percibió que el rendimiento de los alumnos alcanzo un promedio de 17 ; siendo la nota mínima 14 y la máxima 18 en comparación del modelo tradicional que alcanzo en promedio 10,8. Conclusiones: Se pudo demostrar que este modelo ESEAC mejora a plenitud el aprendizaje significativo de los alumnos, así mismo enriquece la actividad evaluadora de los docentes en la medida en que, ademas de proporcionarles todos los datos que habitualmente puedes extraerse de una sección de aprendizaje.

Palabras clave: Estrategias; Aprendizaje significativo; Rendimiento; Evaluación cognitiva

ABSTRACT

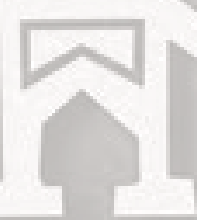

Objective: To propose the cognitive model of evaluation as a resource that enables the improvement of significant learning of students of the Professional School of Education of the National University "Santiago Antunez de Mayolo - Branch Barranca. Material and Methods: A quasi - experimental design was applied, using the analytical method and synthesis, the Scale of Contextualized Learning Strategy (ESEAC) was used as a schema. the sample identified was 40 students divided into 2 groups, use of control and the other experiment. Result: After the application of the instrument it was perceived that the performance of the students reached an average of 17; being the minimum grade 14 and the maximum 18 compared to the traditional model that reached on average 16.8. Conclusions: It was possible to demonstrate that this ESEAC model fully improves the students 'significant learning, as well as enrich the teachers' evaluation activity to the extent that, in addition to proportional all the data that can usually be extracted from a learning section.

Key words: Strategies; significant learnin; performance; cognitive evaluation.

${ }^{1}$ Facultad de Ciencias Sociales, Educación y Comunicación, Universidad Nacional Santiago Antúnez de Mayolo. Email: mamore_08_31@hotmail.com 


\section{INTRODUCCIÓN}

Los sucesivos cambios que se perciben hoy en día en diversos ámbitos de la sociedad: políticos, económicos, sociales, culturales ha hecho posible, en el plano pedagógico, plantear nuevos horizontes con marcos teóricos más completos; así como instrumentos y modelos de evaluación que posibiliten enriquecer el proceso de aprendizaje de los estudiantes.

Somos conscientes de las orientaciones que se ha generado a lo largo del proceso de investigación, por que vienen sugeridas con el convencimiento de que es posible un sustancial enriquecimiento de la evaluación, mediante la creación y utilización de instrumentos más acordes con los logros de la psicología del aprendizaje.

Esta investigación comienza analizando lo que ha sido la teoría y la práctica del aprendizaje, tomando como referencia los planteamientos de las teorías: conductista y cognoscitiva; seguidamente la forma como se lleva cabo la evaluación educativa. En ella debemos destacar los aportes de Delgado (2008), Cid et al. (1991), Díaz y Hernández (1998), Ontoria et al (2005), Pulgar (2005).

La realización del trabajo constituye un gran aporte que servirá de orientación para la implementación de un nuevo sistema de evaluación diferente a lo tradicional y obsoleta que se aplica en la Escuela profesional de Educación de la Filial Barranca; el cual no propicia y garantiza buenos aprendizajes en los estudiantes; quienes, al salir de la Universidad, deben mostrar un buen y alto nivel de competitividad; necesario en todo profesional joven de nuestro tiempo.

Tomando como referencia estos hechos nos formulamos la siguiente interrogante:

¿Cómo influye el modelo cognitivo de evaluación en el mejoramiento del aprendizaje significativo de los alumnos de la Escuela Profesional de Educación de la UNASAM? y llegando al objetivo de determinar la influencia que existe entre el modelo cognitivo de evaluación para contribuir en el mejoramiento del aprendizaje significativo de los alumnos de la escuela profesional de educación de la UNASAMfilial barranca.

\section{MATERIALES Y MÉTODOS}

En la investigación se ha utilizado diversos elementos para la comprobación de la hipótesis. Primero se ha diseñado la Escala de Estrategias de Aprendizaje Contextualizado (ESEAC), que sirvió como matriz para la evaluación comparativa de las sesiones de clase en las diferentes aulas de la Escuela
Profesional de Educación según la muestra. Asimismo, se ha elaborado el Esquema del Modelo Cognitivo, base principal para seguir la secuencia del diseño de clase en la aplicación del grupo experimental.

El estudio es una investigación aplicada de tipo Cuasi Experimental. La población estuvo conformada por 122 estudiantes con características comunes y de los que se deseó conocer las razones de cómo lograr el mejoramiento del aprendizaje significativo. La muestra, conformada por 40 alumnos, entre hombres y mujeres del IV ciclo, grupos A y B de la especialidad de Comunicación, Lingüística y Literatura. Considerada representativa por poseer todas las características de la población. Asimismo, los métodos que se emplearon fueron el análisis, la síntesis y comparación.

Los instrumentos diseñados y empleados para la recolección de información fue la prueba de entrada (PRE TEST) y prueba de salida (POST TEST) aplicados a cada grupo de manera diferencial. Es decir, para el grupo de control se hizo una sesión normal como se viene ejecutando las clases. En cambio, en el grupo experimental se realizó con el esquema del modelo cognitivo.

Una vez recopilados la información para el análisis y procesamiento de la información se hizo uso de la

estadística descriptiva e inferencial.

-


Escala De Estrategia De Aprendizaje RESULTADOS

Contextualizado (ESEAC)

\begin{tabular}{|c|c|c|}
\hline $\mathbf{N}^{\circ}$ & DIMENSIONES & PROCEDIMIENTOS \\
\hline I & $\begin{array}{l}\text { Conocimiento } \\
\text { General del } \\
\text { Tema }\end{array}$ & $\begin{array}{l}\text { - Comprensión } \\
\text { - Planificación } \\
\text { - Ejecución }\end{array}$ \\
\hline II & $\begin{array}{l}\text { Dominio de los } \\
\text { Procesos } \\
\text { Cognitivos }\end{array}$ & $\begin{array}{l}\text { - Lenguaje Verbal } \\
\text { - Lenguaje Icónico } \\
\text { - Lenguaje Analógico }\end{array}$ \\
\hline III & $\begin{array}{l}\text { Calidad del } \\
\text { Razonamiento } \\
\text { Inferencial }\end{array}$ & $\begin{array}{l}\text { - Razonamiento } \\
\text { Deductivo } \\
\text { - Razonamiento } \\
\text { Inductivo }\end{array}$ \\
\hline IV & $\begin{array}{l}\text { Nivel de } \\
\text { abstracción }\end{array}$ & $\begin{array}{l}\text { Grados de abstracción: } \\
\text { - Nivel } 0 \\
\text { - Nivel } 1 \\
\text { - Nivel } 2\end{array}$ \\
\hline V & $\begin{array}{l}\text { Conciencia } \\
\text { Cognitiva o Meta } \\
\text { conocimiento }\end{array}$ & $\begin{array}{l}\text { Percepción del } \\
\text { conocimiento } \\
\text { conceptual } \\
\text { - Procedimiento } \\
\text { estratégico } \\
\text { - Autoevaluación }\end{array}$ \\
\hline
\end{tabular}

Los datos que se muestran a continuación muestran el valor que se debe de asignar al nuevo modelo de evaluación toda vez que posibilita logros significativos en cuanto al aprendizaje de los alumnos.

Tabla 1. Rendimiento académico de los estudiantes del curso de estrategias metodológicas.

\begin{tabular}{cc}
\hline Modelo Cognitivo & Modelo Tradicional \\
\hline $\mathrm{n}_{1}=20$ & $\mathrm{n}_{2}=20$ \\
$\bar{x}_{1}=17.05$ & $\bar{x}_{2}=10.80$ \\
$\mathrm{~S}_{1}=1.146$ & $\mathrm{~S}_{2}=1.989$ \\
Mínimo $=14$ & Mínimo $=6$ \\
Máximo $=18$ & Máximo $=13$ \\
\hline
\end{tabular}

Los datos muestran que utilizando el modelo de evaluación Cognitiva los alumnos tuvieron un rendimiento promedio de 17 siendo la nota mínima 14 y la máxima 18. En comparación a lo que se puede percibir utilizando el modelo tradicional de evaluación donde los alumnos tuvieron un ESQUEMA DIDÁCTICO DEL MODELO rendimiento promedio de 10.8 siendo lanota mínima COGNITIVO

I. Conocimiento Intelectivo del Tema:

1.1. Comprensión integral del tema.

1.2. Planificación o programación del tema

1.3. Elaboración del tema

II. Desarrollo de los Procesos Cognitivos:

2.1. Elaboración de un glosario o categorías conceptuales.

2.2. Elaboración de un grafico que representa el tema.

2.3. Representa el contenido temático mediante la técnica del juego de roles.

III. Razonamiento Lógico del Conocimiento: 6 y la máxima 13.

Tabla 2. Rendimiento académico de los alumnos de educación según el modelo.

\begin{tabular}{|c|c|c|c|c|c|}
\hline & & $N$ & Media & Desviación típ. & $\begin{array}{c}\text { Error típ } \\
\text { de la } \\
\text { media }\end{array}$ \\
\hline Rendimiento & $\begin{array}{l}\text { Modelo } \\
\text { cognitivo }\end{array}$ & 20 & 17.05 & & .256 \\
\hline & $\begin{array}{c}\text { Modelo } \\
\text { Tradicion } \\
\text { al }\end{array}$ & 20 & 10.80 & & .445 \\
\hline
\end{tabular}

Los datos mostrados en la tabla 2 ayudan a validar que el rendimiento académico de los alumnos de Educación es mayor con el Modelo Cognitivo de 3.1. Argumenta el conocimiento teórico à través de la técnica de la exposición.

IV. Niveles de Conceptualización:
4.1. Alto : :3
4.2. Medio : 2
4.3. Regular : 1
4.4. Nada : :0

V. Metaevaluación:

5.1. Autoconciencia.

5.2. Autoevaluación. evaluación que con el Modelo tradicional $(\alpha=0.05)$. 
Prueba de Levene

para la igualdad de varianzas

\begin{tabular}{|c|c|c|c|c|c|c|c|c|c|c|}
\hline & & $\mathrm{F}$ & Sig. & $\mathrm{t}$ & gl & $\begin{array}{c}\text { Sig. } \\
\text { (bilateral } \\
\text { ) }\end{array}$ & $\begin{array}{l}\text { Diferencia } \\
\text { de medias }\end{array}$ & $\begin{array}{c}\text { Error típ. } \\
\text { De la } \\
\text { diferencia }\end{array}$ & $\begin{array}{r}95 \% \\
\text { confia } \\
\text { di } \\
\text { Inferio }\end{array}$ & $\begin{array}{l}\text { tervalo de } \\
\text { tza para la } \\
\text { erencia } \\
\text { Superior }\end{array}$ \\
\hline \multirow{2}{*}{ Rendimiento } & $\begin{array}{l}\text { Se han asumido } \\
\text { varianzas iguales }\end{array}$ & 2.788 & .103 & 12.174 & 38 & .000 & 6.250 & .513 & 5.211 & 7.289 \\
\hline & \multicolumn{2}{|c|}{$\begin{array}{l}\text { No se han asumido } \\
\text { varianzas iguales }\end{array}$} & & 12.174 & 30.357 & .000 & 6.250 & .513 & 5.202 & 7.298 \\
\hline
\end{tabular}

Prueba T para la igualdad de medias

cognitivo de evaluación, ningún alumno demostraba niveles altos en sus capacidades. Pero,

\section{DISCUSIÓN}

La aplicación del nuevo modelo cognitivo de evaluación a través de su esquema didáctico ha permitido comprender que las sesiones de aprendizajes son las que orientan un tratamiento contextualizado, en donde la escala aplicada en relación a este diseño ha demostrado altos coeficientes de fiabilidad y validez; la cual nos permite afirmar que, siguiendo los pasos, el profesor podrá alcanzar una visión muy matizada y rica del proceso de aprender de sus alumnos; entender como aprenden lo que se les ensena; en qué aciertan, fallan y por qué. Con el fin de esclarecer el logro de tales metas, hemos aplicado la Escala de Estrategias de Aprendizaje Contextualizado, acondicionándole a los objetivos trazados en la investigación y determinando las dimensiones; punto central de la estrategia evaluativa.

La sustentación de la ESEAC permite enriquecer la actividad evaluadora de los profesores en la medida en que, además de proporcionarles todos los datos que habitualmente puede extraerse de una sesión de clase, en relación a su rendimiento académico, ofrece una radiografía sobre el perfil del pensamiento estratégico en el que se muestran intuitivamente la secuencia completa de los pasos fundamentales dados por el alumno a lo largo del desarrollo de su proceso de aprendizaje.

Asimismo, la utilización de la ESEAC como nuevo instrumento de evaluación nos ha permitido $\mathrm{y}$ permitirá al profesor asignar toda una selección especial, en donde los alumnos guarden entre si un alto nivel de afinidad referente al pensamiento estratégico; entendido éste como una síntesis del dominio de las dimensiones que tiene el esquema didáctico para su aplicación en una sesión de clase.

Así tenemos que, antes de aplicarse el modelo después de la aplicación del esquema didáctico del modelo, la mayor parte de los alumnos del grupo experimental, lograron niveles altos de rendimiento; el nivel más alto se ubicó entre los promedios del 16 y 18 , resultados ponderados totalmente.

Por otro lado, Baker, $(2000)$, con quien concordamos considera que los diseños cuasi experimentales, conocidos también como aleatorización, son las metodologías de evaluación más sólidas. Al distribuir aleatoriamente la intervención entre los beneficiarios calificados, el proceso de asignación mismo crea grupos de tratamiento y de control comparables que son estadísticamente equivalentes entre sí, a condición de que las muestras sean de tamaño adecuado. Se trata de un resultado muy convincente porque, en teoría, los grupos de control generados mediante asignación aleatoria sirven como un escenario contra factual perfecto, sin los dificultosos problemas de sesgo de selección que existen en todas las evaluaciones y agrega que la principal ventaja de esta técnica es la simplicidad en la interpretación de los resultados, puesto que el efecto del programa sobre el resultado que se evalúa se puede medir a través de la diferencia entre las medias de las muestras del grupo de tratamiento y el grupo de control.

Según los resultados estadísticos, mostrados en la tabla $\mathrm{N}^{\circ} 1$, los estudiantes que participaron en este nuevo modelo de evaluación cognitiva tuvieron un rendimiento promedio superior a 16; así mismo observamos que la mayoría de los alumnos obtuvo 18. En consecuencia, los aprendizajes significativos han mejorado totalmente; lo que se determina que existe influencia entre el modelo cognitivo de evaluación con los aprendizajes significativos de los alumnos y que además contribuye al mejoramiento de sus capacidades. De la misma manera se está dando respuesta al 
problema general de la investigación por que permite entender el grado de influencia en los aprendizajes con la aplicación del esquema didáctico de la ESEAC en una sesión de clase, con lo que se acepta la $\mathrm{H} 1$ en cuestión al progreso del rendimiento académico.

En cambio, a los alumnos en quienes se utilizó el modelo de evaluación tradicional tuvieron un rendimiento promedio de 10.8 siendo la nota mínima 06 y la máxima 13. Así mismo observamos que la mayoría de los alumnos obtuvo 11. Esta evaluación se hizo con el registro auxiliar que se usa actualmente en la Universidad en todas las Facultades y las sesiones de clase la que, regularmente, se ejecutan hasta el día de hoy. Además, se aplicó al grupo de control donde no recibió ningún tratamiento de la ESEAC. Por lo tanto, la prueba estadística arroja un $\mathrm{P}=0.000<$ 0.05 , entonces se rechaza Ho.

\section{CONCLUSIONES}

1. La aplicación del modelo cognitivo de evaluación, mejora a plenitud el aprendizaje significativo de los alumnos de la Escuela Profesional de Educación de la Universidad Nacional "Santiago Antúnez de Mayolo" Filial Barranca.

2. La ESEAC, ha demostrado que es una nueva versión de cómo procesar metodologías de aprendizaje y de evaluación, porque presenta un nuevo modelo de calidad, acorde a las nuevas tendencias de la Psicología Cognitiva del aprendizaje y permite enriquecer la actividad evaluadora de los docentes en la medida e que, además de proporcionarles todos los datos que habitualmente pueden extraerse de una sesión de aprendizaje, ofrece también una radiografía del perfil del pensamiento estratégico, en el que se muestra pormenorizadamente la secuenciă completa de los pasos fundamentales dados por los estudiantes a lo largo del desarrollo de su proceso de aprender o, lo es lo mismo, las estrategias mediante las cuales intenta alcanzar el dominio de lo que aprende desglosando cada uno de las dimensiones que tiene el esquema didáctico de evaluación cognitiva.

3. Aplicando el nuevo modelo de evaluación Cognitiva se tuvo un rendimiento promedio de 17 siendo la nota mínima 14 y la máxima 18. Así mismo observamos que la mayoría de los alumnos obtuvo 18 , en consecuencia, los aprendizajes significativos mejoraron totalmente; lo que se determina la influencia entre el modelo cognitivo de evaluación con los aprendizajes significativos de los alumnos y que además contribuye al mejoramiento de sus capacidades en relación a sus procesos cognitivos. De la misma manera se dio respuesta al problema general de la investigación por que permitió entender el grado de influencia en los aprendizajes con la aplicación del esquema didáctico de la ESEAC en una sesión de clase, con lo que se acepta la $\mathrm{H} 1$ en cuestión al progreso del rendimiento académico.

\section{AGRADECIMIENTO}

Agradecer a la institución que permitio esta investigación.

\section{REFERENCIA BIBLIOGRÁFICA}

Baker, J.L. 2000. Evaluación del impacto de los proyectos de desarrollo en la pobreza: Manual para profesionales. Washington: Banco Mundial.

Cid, Raúl, De la Garza, Eduardo y Ortiz, José. 1991. Evaluación cualitativa en la educación superior: cinco estudios de caso. México: Limusa.

Delgado, Kenneth. 2008. Evaluación en la educación superior. Lima: Universidad Nacional Mayor de San Marcos.

Díaz, Frida. y Hernández, Gerardo. 1998. Estrategias docentes para un aprendizaje significativo. México: Mc Graw Hill.

Ontoria, Antonio, Gómez, Juan y Molina, Ana. 2005. Potenciar la capacidad de aprender a aprender. Madrid: Narcea.

Pulgar, José. 2005. Evaluación del aprendizaje en educación no formal. Madrid: Narcea. 